\title{
Exophthalmos Revealing a Krukenberg Tumor: A Case Presentation and Review of the Literature
}

\author{
Mohamed Fetohi $^{\mathrm{a}} \quad$ Sanaa Errarhay ${ }^{\mathrm{b}} \quad$ Aziz Bazine $^{\mathrm{a}} \quad$ Tarikq Namad $^{\mathrm{a}}$ \\ ${ }^{a}$ Medical Oncology Department, Military Hospital Moulay Ismaïl, Meknès, Morocco; \\ ${ }^{b}$ Department of Gynecology and Obstetrics, University Hospital of Fez, Fez, Morocco
}

\section{Keywords}

Krukenberg tumor $\cdot$ Signet ring cell carcinoma $\cdot$ Ovarian metastases $\cdot$ Ocular metastases

\begin{abstract}
Background: Krukenberg tumor is a well-known ovarian metastasis, usually of signet ring cell carcinoma in female patients. In the literature, there are a few documented cases of ocular metastasis in patients with Krukenberg tumor. Case Presentation: We report the case of a 35-year-old single Moroccan woman. She presented chronic pelvic pain, hematemesis and blurring of vision in the left eye. Clinical examination showed a pelvic mass and an exophthalmos with a divergent strabismus in the left eye. Pelvic and abdominal ultrasound showed a right tissular ovarian mass. Computed tomography (CT) scan of the abdomen and pelvis revealed an ovarian tumor and bone metastasis. Orbital magnetic resonance imaging (MRI) showed a hypertrophy of the left inferior rectus muscle behind the exophthalmos. Stomach endoscopy revealed an ulcerated and protruded mass of the antro-fundic junction. Histopathology and immunohistochemistry showed an infiltration of the gastric mucosa by atypically isolated signet ring cells similar to the tumor cells found in the ovarian histopathological exams. Discussion: Krukenberg tumor is an uncommon metastatic tumor of the ovary. This article provides an overview of the major pathological manifestations of Krukenberg tumor, patient characteristics, clinical and laboratory features of the disease, prognostic factors, and current knowledge about its pathogenesis.




\section{Background}

Metastatic dissemination of gastric signet ring cell carcinoma to the ovaries is referred to as Krukenberg tumor, accounting for $1-2 \%$ of all ovarian tumors. The stomach is the primary site in most Krukenberg tumor cases (70\%). Carcinomas of the colon, the appendix, and the breast are the next most common primary sites. The interval between the diagnosis of a primary carcinoma and the subsequent discovery of ovarian involvement is usually 6 months or less, but longer periods have been reported. In many cases, the primary tumor is very small and can escape detection. A history of a prior carcinoma of the stomach or another organ can be obtained in only $20-30 \%$ of cases [1].

We present a case of a young patient with an occult gastric signet ring cell carcinoma that metastasized to the left ocular and ovarian tissue.

\section{Case Presentation}

We report the case of a 35-year-old single Moroccan woman, with a history of breast cancer in her maternal family. She presented chronic pelvic pain, hematemesis, and right hip pain with 1-week history of blurred vision in the left eye. Clinical examination showed a firm, painless, and ill-defined mass. The patient was referred for ophthalmological evaluation, where an exophthalmos with a divergent strabismus in the left eye was found. The anterior segment and the fundus of the same eye were found to be normal.

Abdominal and pelvic ultrasound showed a right tissular ovarian mass, which measured 140/100 mm. Computed tomography (CT) of the abdomen and pelvis revealed an ovarian tumor with lateral aortic lymph nodes and bone metastasis at the right ischiopubic branch (fig. 1). Orbital magnetic resonance imaging (MRI) showed a hypertrophy of the left inferior rectus muscle behind the exophthalmos. Bone scintigraphy showed exact characteristics of a diffuse metastasis of an ovarian tumor (fig. 2).

Stomach endoscopy revealed an ulcerated and protruded mass of antro-fundic junction, $30 \mathrm{~mm}$ in diameter associated with a hypertrophic fundic fold. Laparotomy revealed a mild abundant serous effusion with a right ovarian mass which was polylobed, solid, and measuring 160/90 mm. Peritoneal carcinomatosis was not found. A right salpingo-oophorectomy with biopsies of the peritoneum and right ovary were performed.

Histopathology and immunohistochemistry showed gastric mucosa infiltrated by atypically isolated signet ring cells, and the result of the salpingo-oophorectomy and biopsy of the right ovary revealed the same tumor cells. The ascitic fluid was inflammatory and negative for malignant cells (fig. 3).

In the multidisciplinary team meeting chemotherapy was recommended, but the patient died before starting chemotherapy.

\section{Discussion}

We have reported a case of Krukenberg tumor with ocular metastasis. Ocular metastasis being the first manifestation of a primary malignant tumor of another organ is not uncommon. Eye symptoms preceded the diagnosis of primary tumor in $31-46 \%$ of patients in two large series [1]. Therefore, a metastatic origin should always be considered when eye lesions of unknown etiology are found. 


\section{Case Reports in Oncology}

Clinical symptoms of Krukenberg tumors are exclusively related to the ovary. Nearly $90 \%$ of patients have symptoms such as abdominal pain and swelling [2]. Some have only gastrointestinal symptoms or remain asymptomatic. Although the frequency is not high, the important symptoms of this tumor are hormone-related such as abnormal genital bleeding or virilization, which are manifestations of hormone production by proliferating ovarian stromal cells within the tumor [3]. In most cases, primary gastric carcinoma and ovarian metastasis are diagnosed concurrently. In $20-30 \%$ of the cases, there is a history of gastric carcinoma when ovarian metastasis is found [4]. In some of these cases, ovarian manifestation occurs years later $[4,5]$. Therefore, it may be prudent to remove the bilateral ovaries of postmenopausal patients with gastric carcinoma. In a small proportion of patients, the primary gastric carcinoma becomes apparent after a significant interval period [6, 7].

Krukenberg tumors are bilateral in more than $80 \%$ of the reported cases, as is the case in our patient. The ovaries are usually asymmetrically enlarged, with a bosselated contour. The sectioned surfaces are yellow or white; they are usually solid, although they are occasionally cystic. Importantly, the capsular surface of the ovaries with Krukenberg tumors is typically smooth and free of adhesions or peritoneal deposits. Other metastatic tumors to the ovary tend to be associated with surface implants. This may explain why the gross morphology of Krukenberg tumor can deceptively appear as a primary ovarian tumor. However, bilateralism in Krukenberg tumor is consistent with its metastatic nature.

Microscopically, Krukenberg tumor has two components: epithelial and stromal. The epithelial component is composed chiefly of mucin-laden signet ring cells with eccentric hyperchromatic nuclei. The cytoplasm of the signet ring cells can be eosinophilic and granular, pale and vacuolated, or it can have a bull's eye appearance containing a large vacuole with a central to paracentral eosinophilic body composed of a droplet of mucin. Mitotic activity is sparse. The signet ring cells can be single, clustered, nested or they can be arranged in tubules, acini, trabeculae, or cords.

The histochemical identification of intracytoplasmic mucin in the signet ring cells is essential for the diagnosis of Krukenberg tumor [8]. Because the intracytoplasmic mucins of the signet ring cells are neutral and acidic, they stain with Mayer's mucicarmine, periodic acid-Schiff with diastase digestion, and Alcian blue stains.

Immunohistochemically, the tumor cells are immunoreactive to epithelial markers, such as cytokeratins (AE1/AE3) and epithelial membrane antigen, and they do not show immunoreactivity to vimentin and inhibin [9].

The mesenchymal component of Krukenberg tumor is of stromal origin and is composed of plump and spindle-shaped cells with minimal cytologic atypia or mitotic activity. Stromal edema can be present focally but is sometimes diffuse and marked to the extent of forming pseudocysts.

It is believed that the majority of cases of Krukenberg tumor metastasize to the ovary via the blood flow. This partly explains the incidence in younger generations whose ovarian blood flow is more abundant than that of older patients [2], as is the case in our patient. There is no evidence, however, that lymphatic spread or intraperitoneal dissemination is not the route of metastasis in Krukenberg tumors. The occurrence of direct lymphatic spread from the stomach may not be plausible, as it means that metastasis occurs against the lymphatic flow. Kakushima et al. [10] thoroughly examined an early diagnosed gastric cancer patient with Krukenberg tumor and found minimal lymphatic invasion and lymph node metastasis at the primary site. They suggested that early lymphatic invasion and subsequent spread into the systemic circulation is an important metastatic pathway of Krukenberg tumor. In addition, Woodruff and Novak [11] reported that 22 of 48 patients with Krukenberg 
tumors had ascites at the time of diagnosis, suggesting that peritoneal spread may be another route of metastasis.

The prognosis of metastatic ovarian cancer is poor. Webb et al. [12] reported that the overall 5-year survival rate of metastatic ovarian tumor cases was $12 \%$ and that of the stomach as a primary site was $5.4 \%$. Generally, ovarian metastases from nongynecological organs have a poor prognosis compared with that from gynecological organs [13].

Kim et al. [14] reported that the extent of the metastatic lesion and residual disease after surgery affected the outcome of the patients, while the size of the ovarian tumor or relapsefree interval was not associated with prognosis.

There is no effective treatment for Krukenberg tumor except for complete surgical resection of both the primary and ovarian lesion. In rare cases where the lesions are confined to the stomach and ovaries, there is a chance for a long survival after surgical treatment [14]. Chemotherapy may be of limited benefit for a minority of patients. As noted above, some surgeons recommend prophylactic oophorectomy as part of the surgical procedure for menopausal or postmenopausal gastric cancer patients [15].

In conclusion, Krukenberg tumor is a metastatic ovarian tumor that is histologically characterized by mucin-laden signet ring cells. The stomach is the most common primary site, but other organs can serve as a primary site. The lymphatic system is the most likely route for metastasis. The prognosis of Krukenberg tumor is poor and no curative treatment is currently available.

\section{Acknowledgements}

The authors' thanks Dr. Rhizlane Belbarakawho was involved in critically revising the manuscript.

\section{Statement of Ethics}

The patient provided written consent for her case to be published.

\section{Disclosure Statement}

The authors declare that they have no competing interests.

\section{References}

1 Holtz F, Hart WR: Krukenberg tumors of the ovary: a clinicopathological analysis of 27 cases. Cancer 1982;50:2438-2447.

2 Young RH, Scully RE: Metastatic tumors of the ovary; in Kurman RJ (ed): Blaustein's pathology of the female genital tract. New York, Springer, 2002, p 1074.

-3 Forest MG, Orgiazzi J, Tranchant DJ: Approach to the mechanism of androgen overproduction in a case of Krukenberg tumor responsible for virilisation during pregnancy. J Clin Endocrinol Metab 1978;47:428.

4 Hale RW: Krukenberg tumor of the ovaries. A review of 81 records. Obstet Gynecol 1968;32:221.

5 Praz V, Nordback P, Billing A: Krukenberg's tumor. Three years after a colic carcinoma. Swiss Surg 2002;8:237. 


\section{Case Reports in Oncology}

\begin{tabular}{l|l}
\hline Case Rep Oncol 2016;9:409-414 \\
\hline $10.1159 / 000448167$ & $\begin{array}{l}\text { (c) 2016 The Author(s). Published by S. Karger AG, Basel } \\
\text { www.karger.com/cro }\end{array}$ \\
\hline
\end{tabular}

Fetohi et al.: Exophthalmos Revealing a Krukenberg Tumor: A Case Presentation and Review of the Literature

6 Joshi VV: Primary Krukenberg tumor of ovary. Review of literature and case report. Cancer 1968;22:1199.

7 Ohara N, Murao S: Development of krukenberg tumor before detection of the primary gastric cancer. Swiss Surg 2002;8:237.

8 Ramzy I: Signet ring stromal tumor of ovary: histochemical, light, and electron microscopic study. Cancer 1976;38:166-172.

9 Wong PC, Ferenczy A, Fan L-D, McCaughey E: Krukenberg tumors of the ovary: ultrastructural, histochemical, and immunohistochemical studies of 15 cases. Cancer 1986;57:751-760.

10 Kakushima N, Kamoshida T: Early gastric cancer with Krukenberg tumor and review of cases of intramucosal gastric cancers with Krukenberg tumor. J Gastroenterol 2003;38:1176.

$\$ 11$ Woodruff JD, Novak ER: The Krukenberg tumor: study of 48 cases from the ovarian tumor registry. Obstet Gynecol 1960;15:351.

12 Webb MJ, Decker DG: Cancer metastatic to the ovary: factors influencing survival. Obstet Gynecol 1975;45:391.

13 Yada-Hashimoto N, Yamamoto T, Kamiura S, Seino H, Ohira H: Metastatic ovarian tumors: a review of 64 cases. Gynecol Oncol 2003;89:314.

14 Kim HK, Heo DS: Pronostic factors of Krukenberg's tumor. Gynecol Oncol 2001;82:105.

15 McGill FM, Ritter DB, Rickard CS, Kaleya RN, Wadler S: Krukenberg tumors: can management be improved? Gynecol Obstet Invest 1999;48:61.

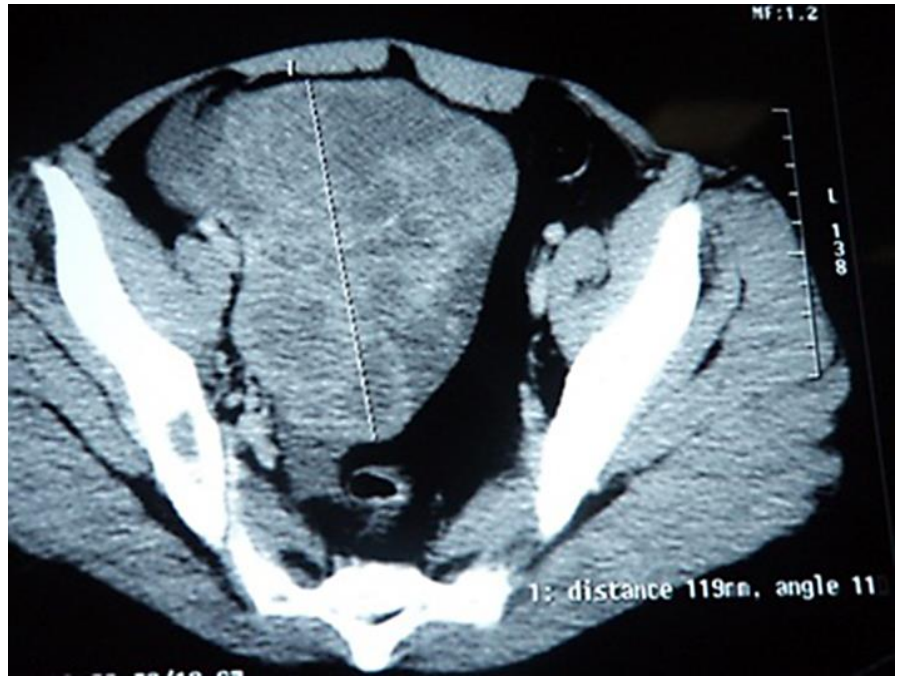

Fig. 1. CT scan of the pelvis: heterogeneous mass of the right ovary, enhanced after contrast material injection. 


\section{Case Reports in Oncology}

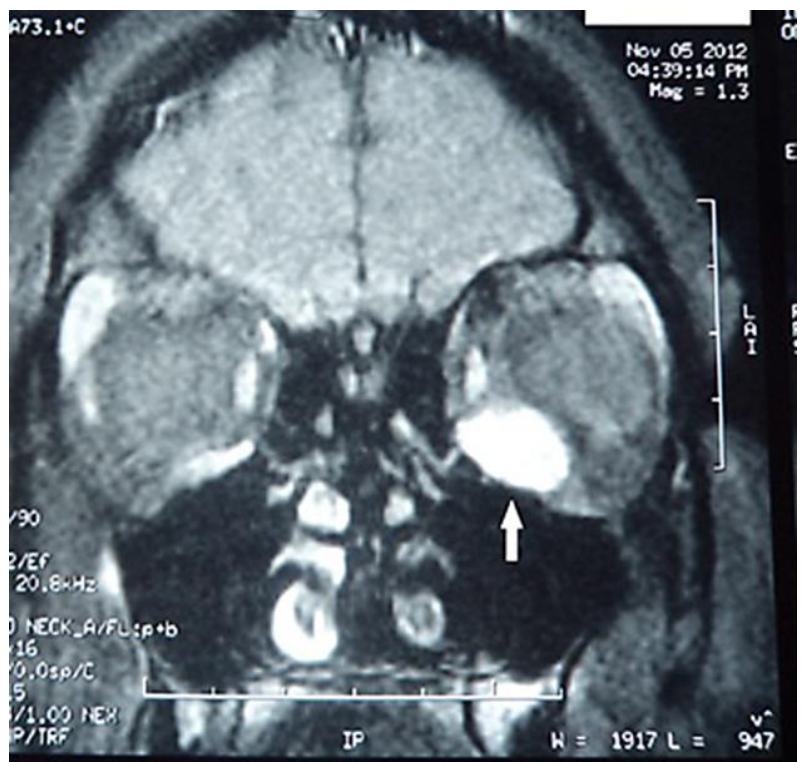

Fig. 2. Coronal view of orbital MRI: hypertrophy of the left inferior rectus muscle.

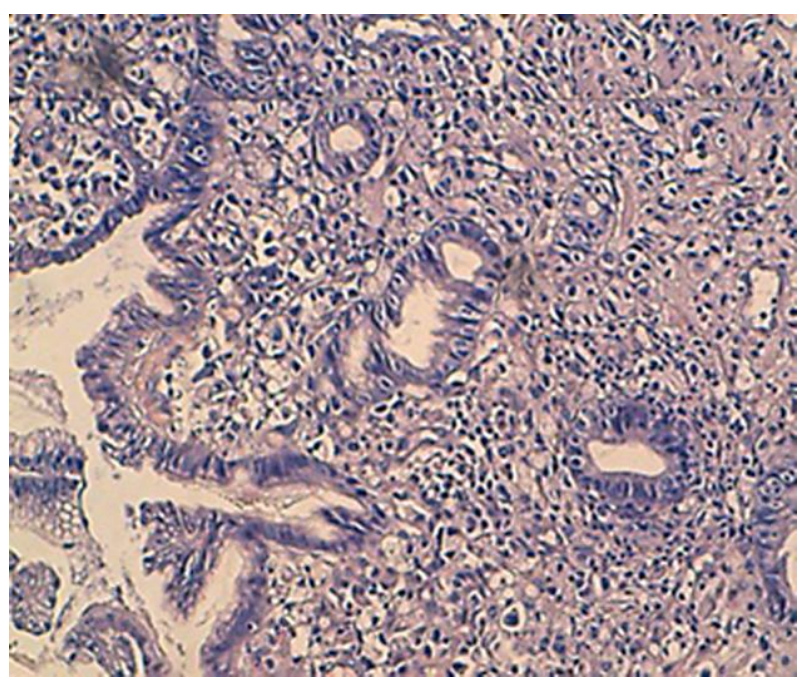

Fig. 3. Gastric mucosa infiltrated by atypically isolated signet ring cells. HES ×200. 